\title{
Unusual Presentation of Iatrogenic Phenytoin Toxicity in a Newborn
}

\author{
Jennifer A. Lowryab, John C. Vandoverc, Jan DeGreeffa, Anthony J. Scalzo a \\ a Cardinal Glennon Regional Poison Center, Saint Louis University School of Medicine, St. Louis, MO \\ b Children's Mercy Hospital, Kansas City, MO \\ c Emergency Medicine/Internal Medicine Residency, Christiana Care Health System, Newark, DE
}

REPRINTED FROM THE INT J MED TOXICOL 2001; 4(1): 1.

\begin{abstract}
Background: Medication errors may produce severe toxicity resulting in hospitalization. This can be compounded if the physician obtains the wrong concentration from a reference manual and a pharmacy miscalculates the conversion. We report a child presenting with ileus, hypothermia and lethargy after receiving supratherapeutic dosing of phenytoin after a concentration miscalculation.

Case Report: A one-month-old infant presented to the Emergency Department with progressive worsening of abdominal distension, diminished activity, and a one day history of difficulty feeding secondary to a decreased level of consciousness. The past medical history was significant for neonatal Group B Strep meningitis with seizures. Among the child's discharge medications was a prescription for phenytoin $(30 \mathrm{mg} / 5 \mathrm{~mL}) 2.5 \mathrm{cc}$ by mouth three times daily. On exam, the child was hypothermic with pink mottled skin, poor responsiveness, prolonged capillary refill, abdominal distension with hypoactive bowel sounds, and a dysconjugate gaze. The Initial phenytoin serum concentration was $91.8 \mathrm{mcg} / \mathrm{mL}$. She was admitted to the PICU and was started on ampicillin and cefotaxime for $\mathrm{R} / \mathrm{O}$ sepsis. Phenytoin was withheld and subsequent serum concentrations revealed an extremely slow elimination (mcg/mL vs. time pair coordinates were 78.2/13.3h; 74.3/62.3h; 43.7/109.6h; 10.8/160.9h) reflecting zero-order kinetics. Post discontinuing antibiotics, phenytoin levels decreased at rates expected. She was discharged after resolution of symptoms. The MD who had written the phenytoin prescription had based it on the Harriet Lane Handbook, $2000 \mathrm{Ed}$. The $30 \mathrm{mg} / 5 \mathrm{~mL}$ formulation has been unavailable in the US for several years. A community pharmacy substituted the $125 \mathrm{mg} / 5 \mathrm{~mL}$ formulation, but miscalculated the dosage to be $1.6 \mathrm{cc}(40 \mathrm{mg})$ tid.

Conclusions: Abdominal distension and ileus may be presenting symptoms in children at toxic phenytoin levels. Ampicillin and cefotaxime may effect the elimination rate of phenytoin at such levels. We report one of the highest phenytoin levels recorded after therapeutic misadventure. Physicians must be aware of inaccuracies in reference manuals that may result in dosing errors.
\end{abstract}

\section{INTRODUCTION}

Medication errors, be they accidental or iatrogenic, may produce severe toxicity resulting in morbidity or mortality. Phenytoin, a commonly used aromatic anticonvulsant agent, continues to produce iatrogenic toxicity (nystagmus, ataxia, confusion, dysrhythmias and seizures) consequent to dosing errors largely resulting from an under-appreciation of its complex pharmacokinetics (i.e., Michaelis-Menten or concentration dependent kinetics) and/or the profound age-dependence in its plasma clearance. Toxicity may occur, in large part, secondary to incorrect dosing. Incorrect dosing may occur at all levels of the health care team. However, toxicity may occur with failure to recognize developmental differences in drug metabolism and potential

Keywords: phenytoin, drug toxicity Corresponding Author: Jennifer Lowry, MD, Division of Clinical Pharmacology and Medical Toxicology, The Children's Mercy Hospital, 2401 Gilham Road, Kansas City, MO 64108. Email: jlowry@cmh.edu 
drug interactions. As phenytoin is one of the most common anticonvulsants prescribed and has a narrow therapeutic index, medical errors with this medication occur often and can be life threatening. We report an infant with iatrogenic phenytoin intoxication from excessive oral dosing who presented with abdominal distension and ileus. The infant was found to have prolonged plasma clearance of phenytoin which may have been due, in part, to unrecognized drug interactions.

\section{CASE PRESENTATION}

A 29-day-old, $3.7 \mathrm{~kg}$ female infant presented to the Emergency Department (ED) 10 days after discharge from the Neonatal Intensive Care Unit (NICU) with progressive worsening of abdominal distension, decreased feeding, and diminished activity. Past medical history was significant for Group B Streptococcal meningitis resulting in seizures. Phenobarbital, carbamazepine, and phenytoin were used in the NICU, and the patient was discharged on phenobarbital and phenytoin as these were found to be most effective. The serum concentrations were within therapeutic range (phenytoin $9.8 \mathrm{mg} / \mathrm{L}$ ) at the time of discharge.

The examination was significant for a rectal temperature of $92^{\circ} \mathrm{F}$, pink mottled skin, poor responsiveness, and a prolonged capillary refill. Other vital signs included a blood pressure of $107 / 72 \mathrm{~mm} \mathrm{Hg}$, a respiratory rate of 32 per minute and a pulse rate of 100 per minute. Her abdominal exam revealed distension (girth $43 \mathrm{~cm}$ at umbilicus) and hypoactive bowel sounds. The neurologic exam was significant for dysconjugate gaze and absent doll's eye movements. Abdominal films (Figure 1) did not confirm obstruction but showed air filled loops of bowel. A lower gastrointestinal water soluble contrast study was normal. An initial phenobarbital plasma level was $38 \mathrm{mg} / \mathrm{L}$ and the phenytoin level was $91.8 \mathrm{mg} / \mathrm{L}$. Other laboratory tests (e.g., hemogram, renal function tests, hepatic transaminases, bilirubin, serum electrolytes, cerebrospinal fluid studies) were normal with the exception of a prolonged prothrombin time of 50 seconds. Ampicillin and ceftriaxone were administered in the ED. Fluid boluses were given to support circulation and the child was admitted to the Pediatric Intensive Care Unit (PICU). An electrocardiogram was normal and the patient was monitored.

Upon arrival to the PICU, the patient was administered ampicillin and cefotaxime, as empiric treatment for sepsis. Her phenobarbital was continued and phenytoin was discontinued. Repeated assessment of serum phenytoin concentrations (Figure 2) revealed an extremely slow elimination (T50\% of 204.1 hours) ${ }^{*}$ with little to no perceptible reduction over an approximate 40 hour period; a pattern reflective of zero-order kinetics. Antibiotics were discontinued at 44 hours consequent to negative blood cultures followed by a reduction in serum phenytoin concentrations at a dramatically increased rate (T50\% of 35.4

* The abbreviation, $\mathrm{T}^{1 / 2}$, is commonly reserved for describing the elimination half-life of a first order process. In the case described above, the authors chose to use "T50\%" to describe the elimination half-life of phenytoin which in overdose follows a zero-order process.

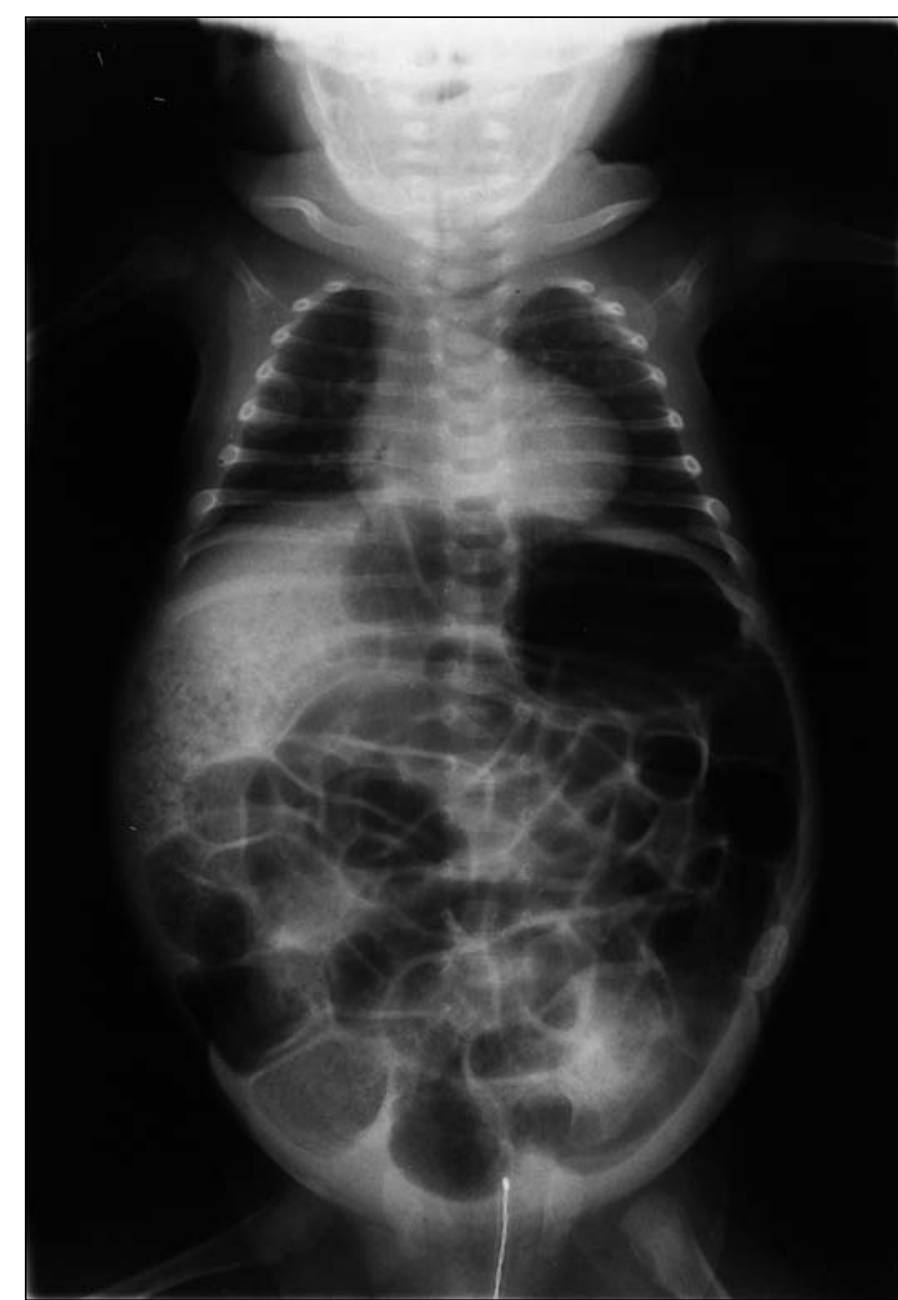

Figure 1. Abdominal radiograph demonstrates prominence of intestinal air.

hours)*. A reduction in abdominal girth was observed on the day following admission and a gradual improvement in the level of consciousness was temporally associated with the reduction in serum phenytoin concentrations. Enteric bacterial and viral cultures were negative as well as a Clostridium difficile toxin assay. Thyroid function studies obtained four days following admission showed a low total thyroxin of $5.4 \mathrm{mcg} / \mathrm{dL}$ (normal range: 6-15.9 mcg/dL) and normal free T4 and TSH. Phenobarbital serum concentrations ranged from 34 to $38 \mathrm{mg} / \mathrm{L}$ throughout her hospitalization course. On the fifth day post admission, the parents noted unusual eye movements described as nystagmoid by housestaff; seizure activity was not witnessed. The patient was discharged after 7 days of hospitalization with complete resolution of her symptoms of phenytoin intoxication.

Re-examination of the patient history revealed that phenytoin intoxication in this infant resulted from a "compound" iatrogenic error. The phenytoin prescription at discharge was written as "phenytoin $(30 \mathrm{mg} / 5 \mathrm{ml}) 2.5 \mathrm{cc}(15 \mathrm{mg})$ po tid" per information from The Harriet Lane Handbook 2000 edition. As the 


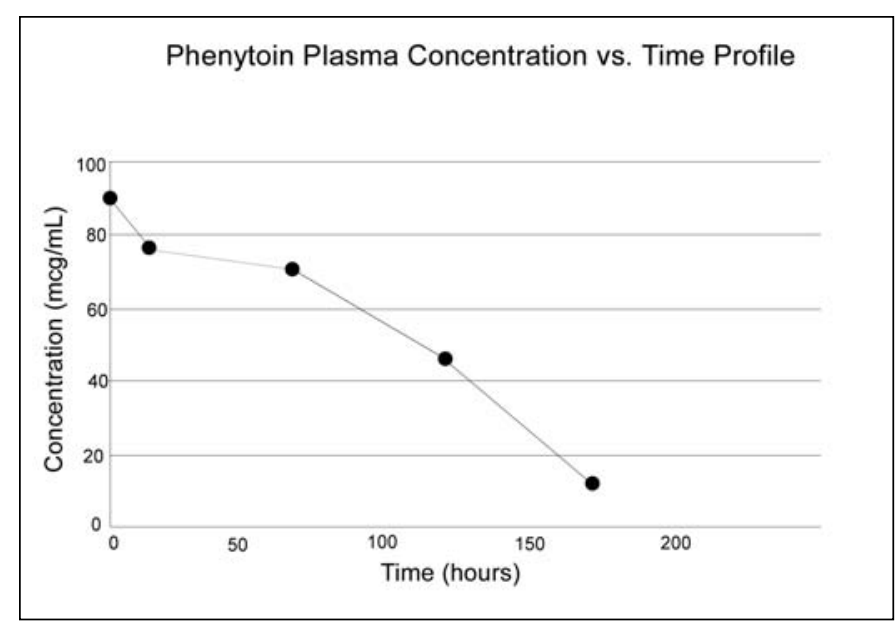

Figure 2. Graph of patient's plasma phenytoin concentration vs. time.

$30 \mathrm{mg} / 5 \mathrm{ml}$ oral suspension formulation has been unavailable in the United States for several years, a community pharmacy substituted the $125 \mathrm{mg} / 5 \mathrm{ml}$ concentration of phenytoin oral suspension and incorrectly calculated the dose to be $1.6 \mathrm{ml}(40 \mathrm{mg})$ tid.

\section{DISCUSSION}

Medication errors may produce severe toxicity resulting in hospitalization with attendant increased morbidity and mortality. Errors are possible at any step of the process from medication selection and ordering, order transcription, drug formulation, and drug dispensing to drug administration. Recent investigations have shown that an error occurs once in every 20 orders for medications. The most common reason for errors is incorrect dosing [1].

In 1998, a letter was written to the editors of Pediatrics informing practitioners of a case of phenytoin toxicity attributable in part to an error in 1996 edition of The Harriet Lane Handbook that documented the availability of the $30 \mathrm{mg} / 5 \mathrm{ml}$ oral suspension formulation. The authors basic premise was to inform physicians that this concentration is not commercially available so as not to repeat the toxicity documented in their patient [2]. However, the reference manual used by the physician in the case above was the 2000 edition of The Harriet Lane Handbook.

Phenytoin toxicity occurs frequently with the usual central nervous system presentation of nystagmus, ataxia, slurred speech, decreased coordination, and seizures. Cardiovascular toxicity has been associated with the intravenous form of phenytoin, but has not been seen with the oral form [3]. Phenytoin toxicity may result from hypothyroidism as decreased metabolism may result. However, as phenytoin is a known hepatic enzyme inducer, it may effect the metabolism and serum concentrations of thyroid hormones [4-6]. Gastrointestinal abnormalities associated with phenytoin toxicity have not been documented in the literature. However, gastrointestinal abnormalities have been associated with hypothyroidism $[7,8]$. Thus, the patient's initial reduction in gastrointestinal motility may be an indirect effect of phenytoin therapy consequent to a lowering of thyroxin levels. Alternatively, it is possible that this "expected" adverse effect may have been worsened by acute phenytoin toxicity with direct effects on gastrointestinal smooth muscle.

The biotransformation of phenytoin to its major hydroxylated metabolite is saturable at plasma concentrations $>5 \mathrm{mg} / \mathrm{L}$ giving rise to a non-linear dose-serum concentration relationship [9]. In neonates and infants to 3 months of age, the free fraction of phenytoin in plasma may be significantly increased (i.e., 10 to $15 \%$ ) over that observed in healthy young adults (e.g., free fraction of 2 to $5 \%$ ) [10]. Consequent to developmental differences (i.e., reductions) in the activity of the rate-controlling enzymes for phenytoin biotransformation (CYP2C9 and CYP3A4) in neonates and very young infants, the increased free fraction of phenytoin produces an increase in the amount of free drug in the plasma and tissue (e.g., CNS) "compartments" [11]. Thus, young infants are predisposed to phenytoin toxicity consequent to developmental differences in drug protein binding and metabolism. Furthermore, concomitant use of medications, such as antibiotics, can effect the pharmacokinetics of phenytoin. While some antibiotics may alter the metabolism of phenytoin, sulfonamides and penicillins can bind to serum proteins causing displacement of phenytoin and increasing the free fraction and clinical symptoms of toxicity [12-19]. This may account for the change in pharmacokinetics (i.e., T50\%) in the case presented. As a result of the relatively low plasma protein binding of cefotaxime (e.g., 30-50\%), it is unlikely that this antibiotic played a role in producing phenytoin toxicity in the infant reported.

\section{CONCLUSIONS}

Phenytoin toxicity may present with gastrointestinal symptoms (e.g., abdominal distension, ileus) mimicking bowel obstruction and may be indistinguishable from gastrointestinal signs and symptoms of hypothyroidism. Developmental reductions in the activity of enzymes responsible for phenytoin biotransformation and in both the affinity and capacity of plasma proteins capable of binding the drug can accentuate toxicity in neonates and the very young (i.e., $<3$ months of age) infants. Penicillins and other antibiotics with significant (i.e., $>70 \%$ ) binding to plasma albumin may potentiate phenytoin toxicity in young infants via competitive inhibition of binding. Physicians and pharmacists need to be aware of the unavailability of the $30 \mathrm{mg} / 5 \mathrm{ml}$ concentration of phenytoin oral suspension formulation that is referenced in the 2000 edition of The Harriet Lane Handbook and also, the ageappropriate dose recommendations of this drug for young infants.

\section{REFERENCES}

1. Anonymous. American Academy of Pediatrics: Committee on Drugs and Committee on Hospital Care. Pediatrics.

1998; $102: 428$. 
2. Sharfstein J, Agrawal D, Jeffrey P. Letter to the editor. Pediatrics. 1998; $102: 1223$.

3. Wyte C, Berk WA. Severe oral phenytoin overdose does not cause cardiovascular mortality. Ann Emerg Med. 1991;20:508.

4. Nation RL, Evans AM, Milne RW. Pharmacokinetic drug interactions with phenytoin (Part II). Clin Pharmacokinet.

1990; $18: 131$.

5. Sarich T, Wright JM. Hypothyroxinemia and phenytoin toxicity: A vicious circle. Drug Metabol Drug Interact.

1996; 13:155.

6. Zhu SQ, Liu XM, Ruan XZ, Cai Z. Changes of thyroid hormone levels in epileptic patients. J Tongji Med Univ.

1994; 14:119.

7. Bassotti G, Pagliacci MC, Nicoletti I, Pelli MA, Morelli A. Intestinal pseudo-obstruction secondary to hypothyroidism. Importance of small bowel manometry. J Clin Gastroenterol. 1992; $14: 56$.

8. Goto S, Billmire DF, Grosfeld JL. Hypothyroidism impairs colonic motility and function. An experimental study in the rat. Eur J Pediatr Surg. 1992;2:16.

9. Battino D, Estienne M, Avanzini G. Clinical pharmacokinetics of antiepileptic drugs in paediatric patients. Clin Pharmacokinet. 1995;29:341.

10. Painter MJ, Minnigh MB, Gaus L, Scher M, Brozanski B, Alvin J. Neonatal phenobarbital and phenytoin binding profiles. J Clin Pharmacol. 1994;34:312.

11. Leeder JS, Kearns GL. Pharmacogenetics in pediatrics. Implications for practice. Pediatr Clin North Am. 1997;44:55.
12. Bachmann K, Schwartz JI, Forney RB, Jaurgeui L. Single dose phenytoin clearance during erythromycin treatment. Research Communications in Chemical Pathology and Pharmacology. 1984;46:207.

13. Tucker RM, Denning DW, Hanson LH, Rinaldi MG, Graybill JR, Sharkey PK, et al. Interaction of azoles with rifampin, phenytoin, and carbamazepine: In vitro and clinical observations. Clin Infect Dis. 1992;14:165.

14. Rose JQ, Choi HK, Schentag JJ, Kinkel WR, Jusko WJ. Intoxication caused by interaction of chloramphenicol and phenytoin. JAMA. 1977;237:2630.

15. Bint AJ, Burtt I. Adverse antiobiotic drug interactions. Drugs. 1980;20:57.

16. Chandra RS, Dalvi SS, Powar HS, Karnad PD, Kshirsagar NA. Phenytoin toxicity due to rifampicin induced hepatic dysfunction. JAPI. 1993;41:536.

17. Dasgupta A, Dennen DA, Dean R, McLawhorn RW. Displacement of phenytoin from serum protein carriers by antibiotics: Studies with ceftriaxone, nafcillin, and sulfamethoxazole. Clin Chem. 1991;37:98.

18. Dasgupta A, Sperelakis A, Mason A, Dean R. Phenytoinoxacillin interactions in normal and uremic sera. Pharmacother. 1997; $17: 375$.

19. Arimori K, Nakano M, Otagiri M, Uekama K. Effects of penicillins on binding of phenytoin to plasma proteins in vitro and in vivo. Biopharm Drug Dispos. 1984;5:219-27. 